\title{
Research progress of circRNA as a biomarker of sepsis: a narrative review
}

\author{
Lei Qi ${ }^{1,2 \#}$, Yan Yan ${ }^{1 \#}$, Bairong Chen ${ }^{3}$, Jiling $\mathrm{Cao}^{1}$, Guiwen Liang ${ }^{1}$, Pan $\mathrm{Xu}^{1}$, Yue Wang ${ }^{1}$, Yuting Ren ${ }^{1}$, \\ Guomin Mao ${ }^{1}$, Zhongwei Huang ${ }^{1}$, Cheng Xu ${ }^{4}$, Haiyan Jiang ${ }^{1}$
}

${ }^{1}$ Department of Emergency Medicine, Affiliated Hospital of Nantong University, Nantong, China; ${ }^{2}$ Rugao Branch Institute, Affiliated Hospital of Nantong University, Nantong, China; ${ }^{3}$ Department of Medical Laboratory, School of Public Health, Nantong University, Nantong, China; ${ }^{4}$ Department of Medical Imaging, Affiliated Hospital of Nantong University, Nantong, China

Contributions: (I) Conception and design: H Jiang, Z Huang; (II) Administrative support: Z Huang, C Xu; (III) Provision of study materials or patients: B Chen, Y Ren; (IV) Collection and assembly of data: G Liang, P Xu; (V) Data analysis and interpretation: G Mao, L Qi; (VI) Manuscript writing: All authors; (VII) Final approval of manuscript: All authors.

\#These authors contributed equally to this work.

Correspondence to: Haiyan Jiang; Zhongwei Huang. Department of Emergency Medicine, Affiliated Hospital of Nantong University, 20 West Temple Road, Nantong 226001, China. Email: jhy@ntu.edu.cn; hzw889@163.com; Cheng Xu. Department of Medical Imaging, Affiliated Hospital of Nantong University, Nantong, China. Email: tdfyxuc@163.com.

Objective: Explore the possibility of circRNAs as markers of sepsis.

Background: Sepsis is an abnormal immune response of our body to infection that can lead to organ failure and death. Although the research on sepsis has been extensive in the past few years, sepsis-associated morbidity and mortality are still increasing. Early diagnosis and early treatment are important for patients with sepsis. Although many markers, including procalcitonin and C-reactive protein, have been proposed as diagnostic indicators of sepsis, there are still challenges in the early diagnosis and treatment of sepsis due to the lack of sensitivity and specificity of these substances. Recently, a large number of studies have found that circular RNAs (circRNAs) participate in a variety of biological functions, such as immune response, regulating the expression of miRNAs, and they are closely related to the occurrence and development of many diseases, including sepsis. However, the clear mechanism of the role of circRNAs has not been fully elucidated. An increasing number of studies have confirmed that circRNAs have potential in the diagnosis and treatment of sepsis. By studying the regulatory mechanism of circRNAs in sepsis, we can search for new molecular intervention targets for the treatment of sepsis, which is conducive to the development of new molecular therapeutic drugs for sepsis.

Methods: In the present study, we summarize and analyze the role of circRNAs in the pathogenesis of sepsis and discuss the possibility of circRNA as a biomarker for the diagnosis of sepsis.

Conclusions: The biological characteristics of circRNAs and their role in the occurrence and development of sepsis make them possible markers of sepsis.

Keywords: Circular RNAs (circRNAs); marker; sepsis

Submitted Feb 23, 2021. Accepted for publication Apr 17, 2021.

doi: 10.21037/atm-21-1247

View this article at: http://dx.doi.org/10.21037/atm-21-1247

^ ORCID: 0000-0002-2237-0577. 


\section{Introduction}

Sepsis is an organ dysfunction formed by the abnormal response of the body to various infections that threaten life (1). In 2017, the global age-standardized mortality rate for sepsis was 148.1 [95\% uncertainty interval (UI) 136.4-161.0] per 100,000 population (2). Although research on sepsis is increasing, sepsis is still a global problem. The pathogenesis of sepsis is complex and includes inflammatory response imbalance, immune dysfunction, mitochondrial damage, coagulation disorders, endoplasmic reticulum stress, autophagy, and other pathophysiological processes, resulting in organ dysfunction (3). At present, some biomarkers used for the clinical diagnosis of sepsis, such as interleukin-6, C-reactive protein, and procalcitonin, have low specificity and sensitivity, the severity and recovery of sepsis cannot be well judged, nor can it be well used in clinical practice (4). Therefore, finding suitable biomarkers is important for the diagnosis and treatment of sepsis.

Circular RNAs (circRNAs) were first discovered in viruses in the 1970 (5). With the development of bioinformatics techniques, such as high-throughput sequencing, more and more circRNAs have been found in a variety of cells. Increasingly, studies have found that circRNAs play an important role in gene transcription (6). CircRNAs participate in a variety of cellular events, including proliferation, differentiation, autophagy, and apoptosis (7), and are closely related to sepsis.

CircRNAs have become a research hotspot, and their occurrence and development are related to a variety of diseases. Because of the above reasons and their biological characteristics, circRNAs have been suggested as a biomarker of sepsis (8). CircRNAs have potential in the diagnosis and treatment of sepsis. In the present study, we review the research progress of circRNA as a biomarker of sepsis and analyze its development in the diagnosis and treatment of sepsis.

We present the following article in accordance with the Narrative Review reporting checklist (available at http:// dx.doi.org/10.21037/atm-21-1247).

\section{Overview of circRNAs}

CircRNAs are generally formed by cyclization of special mRNA through classical splicing and reverse splicing, with a length of about 100 nucleotides (9). Some recent studies have shown that a canonical splicing mechanism produces covalently closed circRNAs through a process called reverse splicing. Different from common linear RNAs (including 5' and $3^{\prime}$ ends), circRNAs have a closed ring structure, no 5' and 3 ' ends, are not affected by RNA exonuclease.

The circRNAs have some biological characteristics like structural stability, higher expression (10), tissue specificity, and high conservation in different species (11). CircRNAs have multiple modes, such as intron pairing driven cyclization, RNA binding protein pairing driven cyclization, and lasso driven cyclization (12). According to the origin of biogenesis, circRNAs can be divided into the following four types: exon circRNAs, intron circRNAs, exon-intron circRNAs, and intergenic circRNAs (13).

In our in-depth study of RNAs, a large number of circRNAs were found to have many important biological functions, such as being miRNA molecular sponge to regulate the concentration of miRNA in body fluids, regulating cell gene transcription and translation, participating in regulating protein translation, and acting as a protein sponge to regulate protein content in cells (14). At the same time, circRNAs are also involved in the immune response of organisms and the regulation of immune diseases (15). The half-life of circRNAs are longer than that of homologs and is more stable (16), and circRNAs act as an miRNA sponge (17). These biological functions and characteristics indicate the potential of circRNAs as markers of sepsis.

\section{Relationship between circRNAs and sepsis}

Although there has been very little study on circRNAs as an indicator of sepsis, it is considered that circRNA has the potential to be a biomarker of sepsis because of its characteristics and its role in the pathogenesis of sepsis.

CircRNAs are involved in the regulation of several different mechanisms in the occurrence and development of sepsis. First, circRNAs act as miRNA sponges to suppress the function of miRNA (18). Second, circRNAs regulate inflammation by participating in the release of inflammatory mediators and regulating the expression of inflammatory cytokines (19). Third, circRNAs regulate cellular immunity by mediating immunosuppressive mediators (20). Fourth, circRNAs are involved in blood coagulation in sepsis and produce thrombus that changes vascular homeostasis. Fifth, circRNAs are involved in gene transcription and regulation. Sixth, circRNAs act as protein sponges to regulate protein release. Seventh, circRNAs cause mitochondrial damage by activating NF- $\mathrm{BB}$ (nuclear factor Kappa-B) and regulating mPTP (mitochondrial permeability transition pore) 


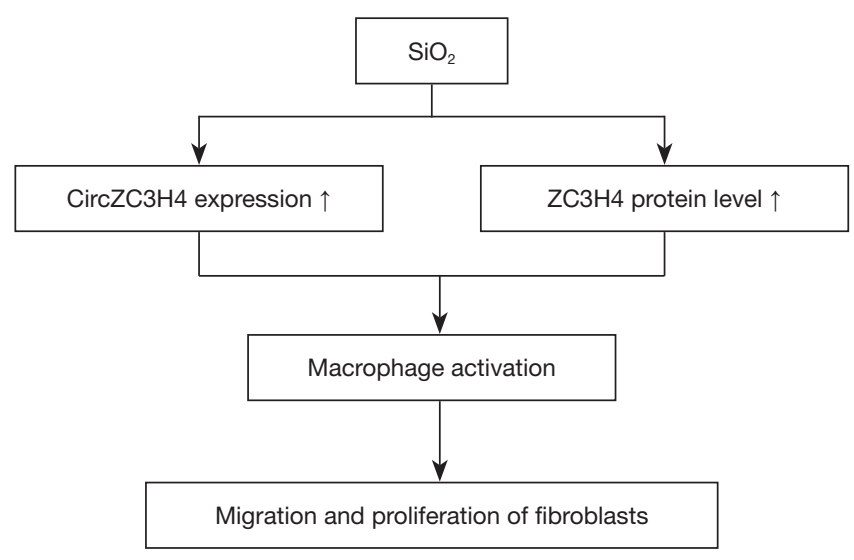

Figure 1 The function of circZC3H4 RNA in the inflammatory model of pulmonary macrophages induced by $\mathrm{SiO}_{2}$.

opening (21).

Bao et al. found that 11 and 126 circRNAs were significantly upregulated and downregulated in septic mice compared with controls, respectively $(\mathrm{P} \leq 0.05$, fold change $\geq 2$ ), and that differentially expressed circRNAs had a high multiple change (fold change $>4, \mathrm{P}<0.05)(22)$. Nie et al. found that compared with the control group, there were 373 up-regulated circRNAs and 428 down-regulated circRNAs in the aortic tissues of animals in the sepsis group, while there were 2,063 up-regulated and 2,903 down-regulated mRNAs in the sepsis group (23).

Because circRNAs can regulate a variety of different molecular mechanisms, including inflammation and immune response, as well as multiple biological processes in a variety of metabolic organs (24), they may play a key role in the occurrence and development of sepsis.

\section{CircRNAs as miRNA sponges}

MiRNAs are small, evolutionarily conserved RNA molecules that are widely distributed in the human body; they can regulate about $30 \%$ of protein-coding genes (25). Due to their important role in the regulation of gene expression, there is great interest in the regulatory mechanisms that can regulate the function of miRNA.

With the continuous progress of various studies, it has been found that circRNAs have miRNA response elements, thus circRNAs have the ability to bind miRNA molecules to regulate miRNA activity (26). This activity is often referred to as the molecular sponge effect of circRNAs. Because miRNAs target multiple coding RNAs,
circRNAs as molecular sponges will affect the expression of multiple genes (22). An increasing number of studies $(27,28)$ have shown that circRNAs play an important role in the regulation of gene expression, partly because they inhibit the activity of miRNA, and this biological funtion of inhibiting activity is mainly realized by acting as a molecular sponge for binding to miRNAs.

\section{Role of circRNAs in inflammation}

The obstructive response of the host to various invasive molecules leads to the imbalance of inflammation, which is the most important basis in the pathogenesis of sepsis and is involved in the entire process of sepsis.

In sepsis, the initial acute response of the host to invasive pathogens can lead to a cytokine storm (29), followed by a cytokine storm that leads to the production of pro-inflammatory cytokines, such as interleukin-1, interleukin- 6 , tumor necrosis factor- $\alpha$, interferon regulatory factor 7 , and activating protein-1, to mediate the initial proinflammatory phase $(30,31)$. MiRNAs can also regulate these key cytokines expressed during septicemia, as well as other pro-inflammatory and anti-inflammatory cytokines that are differentially expressed in sepsis. CircRNAs may regulate the related inflammatory pathway by regulating miRNA.

Yang et al. found that RNA plays an important role in the inflammatory model of pulmonary macrophages induced by $\mathrm{SiO}_{2}(19)$. This study analyzed the molecular mechanism and function of circRNAs in the process of apoptosis, proliferation, and migration were analyzed. The results are shown in Figure 1.

The findings of the present study indicate that there is a relationship between the activation of macrophages induced by $\mathrm{SiO}_{2}$ and the circZC3H RNA/ZC3H4 pathway, and demonstrates that circRNAs are involved in the inflammatory response of pulmonary macrophages in the pathological process of silicosis.

It has been found that circRNAs have the potential to inhibit the function of miRNAs related to M2 polarization of lung macrophages after sepsis-induced lung injury, and it can be speculated that down-regulation of these miRNAs can promote M1 polarization of lung macrophages (22).

In addition, Zhang et al. found that 12 circRNAs were upregulated and 2 circRNAs were downregulated in patients with SLE(Systemic Lupus Erythematosus) (32). The findings of their study confirmed that the downregulation of hsa 
circ_0012919 not only increased the expression of DNMT1 and decreased the expression of CD70 and CD11a but also reversed the deficiency of DNA methylation of CD11a and CD70 in SLE CD4 ${ }^{+} \mathrm{T}$ cells, and reversed DNMT1 through downregulation. The abnormal expression of circ_0012919 in $\mathrm{CD}^{+} \mathrm{T}$ cells was found to support the role of circRNAs in mediating inflammation.

\section{Role of circRNAs in immunosuppression}

Although there is a stress response in the immune system in the early stage of sepsis, immunosuppression may occur at the end of this stage, which seriously affects the survival of patients (3).

Xiong et al. found that circMAN2B2 was highly expressed in glioma tissues and cells, and they found that circMAN2B2 regulates the expression of S100A8 by inhibiting miR-1205, and knockout circMAN2B2 can increase the expression of miR-1205 (33). S100A8 is an important protein related to immunosuppression in sepsis $(34,35)$.

Circ_0005075 participates in the regulation of miR-23a$5 \mathrm{p}$ and miR-23b-5p23 (26). The expression of miR-23a-5p is upregulated during sepsis, which leads to acute respiratory distress syndrome induced by lipopolysaccharides (36). The activation of myocardial fibrosis by miR-23b is a key factor in the development of myocardial dysfunction in the late sepsis. At the same time, miR-23b inhibitor can reduce cardiac fibrosis in advanced sepsis. Blocking the expression of miR-23b may be an effective method to prevent septicemia induced cardiac dysfunction (37). This suggests that circ_0005075 may play a role in the treatment of sepsis.

\section{Role of circRNAs in coagulation dysfunction}

Under normal physiological conditions, the activation of the coagulation system is regulated by the following three important physiological anticoagulation pathways: the tissue factor pathway inhibition system, the activated protein C system, and the antithrombotic system; these systems regulate the systematic activation of clotting (38). All three pathways show a certain degree of disorder during sepsis.

Coagulation dysfunction is an important clinical manifestation in the process of sepsis. Endothelial cells play a key role in maintaining vascular homeostasis and activating different physiological and pathological processes of the blood system, such as thrombosis, inflammation, and vascular remodeling (39). Chen et al. found that endothelial cell apoptosis can worsen sepsis (40).

Cheng et al. found that knockout hsa_circ_0068087 inhibits human umbilical vein endothelial cells (HUVEC) dysfunction and inflammation, which is induced by high glucose (HG), by inhibiting the TLR4 (toll-like receptor 4)/NF- $\kappa$ B/NLRP3 (NOD-, LRR- and pyrin domaincontaining 3) inflammatory signal pathway (41). Under the condition of HG, the downregulation of miR-197 reverses HUVEC dysfunction and inflammation caused by the silencing of hsa_circ_0068087.

Therefore, we can speculate that the abnormal expression of circRNAs will lead to endothelial function, and even coagulation dysfunction, and ultimately affect the occurrence and development of sepsis.

\section{CircRNAs are involved in host resistance to viruses}

There only a few studies on the involvement of circRNAs in viruses resistance, but there are also related results.

Li et al. demonstrated that the double-stranded RNA domain containing the immune factor NF90/NF110 is a key regulator of circRNA biogenesis (42). NF90/NF110 promotes the production of annular RNA in the nucleus by combining with intronic RNA pairs that juxtapose circRNAs to form exon(s); and the NF90/NF110 also interact with mature circRNAs in the cytoplasm. When the host is infected with the virus, the expression of circRNAs decreases, in part because the nucleus exports NF90/NF110 to the cytoplasm. At the same time, NF90/NF110 that is released from the circRNA complex binds to viral mRNA as part of its antiviral immune response. This indicates that circRNAs are involved in the antiviral process of the host.

\section{Summary}

The pathogenesis of sepsis is extremely complex. Understanding the molecular mechanism in the occurrence and development of the disease is the only way to understand its biomarkers and specific treatments. Increased research on circRNAs provides a new direction to diagnose and treat sepsis. With increasing studies on circRNAs, we have reason to believe that circRNAs will become a hotspot of sepsis research, and these researches will have a broad development prospect.

Other biological characteristics of circRNAs, such as tissue specificity, stability in blood, and relatively long halflife, not only make it a biomarker but also a new target for 
the diagnosis and treatment of sepsis.

At present, the research on the formation, classification, characteristics, and biological functions of circRNAs are in the initial stage. There is no corresponding clinical data, and a large number of clinical samples are needed for detection and analysis. The expression and regulation of circRNA genes and their role in the occurrence and development of sepsis warrant further study.

\section{Acknowledgments}

Funding: This study was supported by the National Natural Science Foundation of China Youth (No. 81801893), the Nantong Clinical Medicine Research Center (Nos. HS2019005 and HS2020001), and the Nantong Scientific Projects (Nos. MS12020006 and MS12020017).

\section{Footnote}

Reporting Checklist: The authors have completed the Narrative Review reporting checklist. Available at http:// dx.doi.org/10.21037/atm-21-1247

Conflicts of Interest: All authors have completed the ICMJE uniform disclosure form (available at http://dx.doi. org/10.21037/atm-21-1247). The authors have no conflicts of interest to declare.

Ethical Statement: The authors are accountable for all aspects of the work in ensuring that questions related to the accuracy or integrity of any part of the work are appropriately investigated and resolved.

Open Access Statement: This is an Open Access article distributed in accordance with the Creative Commons Attribution-NonCommercial-NoDerivs 4.0 International License (CC BY-NC-ND 4.0), which permits the noncommercial replication and distribution of the article with the strict proviso that no changes or edits are made and the original work is properly cited (including links to both the formal publication through the relevant DOI and the license). See: https://creativecommons.org/licenses/by-nc-nd/4.0/.

\section{References}

1. Singer M, Deutschman CS, Seymour CW, et al. The Third International Consensus Definitions for Sepsis and Septic Shock (Sepsis-3). JAMA 2016;315:801-10.
2. Rudd KE, Johnson SC, Agesa KM, et al. Global, regional, and national sepsis incidence and mortality, 1990-2017: analysis for the Global Burden of Disease Study. Lancet 2020;395:200-11.

3. Huang M, Cai S, Su J. The Pathogenesis of Sepsis and Potential Therapeutic Targets. Int J Mol Sci 2019;20:5376.

4. Pierrakos C, Vincent JL. Sepsis biomarkers: a review. Crit Care 2010;14:R15.

5. Sanger HL, Klotz G, Riesner D, et al. Viroids are singlestranded covalently closed circular RNA molecules existing as highly base-paired rod-like structures. Proc Natl Acad Sci U S A 1976;73:3852-6.

6. Salzman J. Circular RNA Expression: Its Potential Regulation and Function. Trends Genet 2016;32:309-16.

7. Haque S, Harries LW. Circular RNAs (circRNAs) in Health and Disease. Genes (Basel) 2017;8:353.

8. Zhang TN, Li D, Xia J, et al. Non-coding RNA: a potential biomarker and therapeutic target for sepsis. Oncotarget 2017;8:91765-78.

9. Altesha MA, Ni T, Khan A, et al. Circular RNA in cardiovascular disease. J Cell Physiol 2019;234:5588-600.

10. Chen XT, Li ZW, Zhao X, et al. Role of Circular RNA in Kidney-Related Diseases. Front Pharmacol 2021;12:615882.

11. Barrett SP, Salzman J. Circular RNAs: analysis, expression and potential functions. Development 2016;143:1838-47.

12. Ashwal-Fluss R, Meyer M, Pamudurti NR, et al. circRNA biogenesis competes with pre-mRNA splicing. Mol Cell 2014;56:55-66.

13. Huang C, Shan G. What happens at or after transcription: Insights into circRNA biogenesis and function. Transcription 2015;6:61-4.

14. Li X, Yang L, Chen LL. The Biogenesis, Functions, and Challenges of Circular RNAs. Mol Cell 2018;71:428-42.

15. $\mathrm{Ng}$ WL, Marinov GK, Liau ES, et al. Inducible RasGEF1B circular RNA is a positive regulator of ICAM1 in the TLR4/LPS pathway. RNA Biol 2016;13:861-71.

16. Enuka Y, Lauriola M, Feldman ME, et al. Circular RNAs are long-lived and display only minimal early alterations in response to a growth factor. Nucleic Acids Res 2016;44:1370-83.

17. Panda AC. Circular RNAs Act as miRNA Sponges. Adv Exp Med Biol 2018;1087:67-79.

18. Hansen TB, Jensen TI, Clausen BH, et al. Natural RNA circles function as efficient microRNA sponges. Nature 2013;495:384-8.

19. Yang $X$, Wang J, Zhou Z, et al. Silica-induced initiation of circular ZC3H4 RNA/ZC3H4 pathway promotes 
the pulmonary macrophage activation. FASEB J 2018;32:3264-77.

20. Chen X, Yang T, Wang W, et al. Circular RNAs in immune responses and immune diseases. Theranostics 2019;9:588-607.

21. Zhao X, Ma X, Guo J, et al. Circular RNA CircEZH2 Suppresses Transmissible Gastroenteritis Coronavirusinduced Opening of Mitochondrial Permeability Transition Pore via Targeting MiR-22 in IPEC-J2. Int J Biol Sci 2019;15:2051-64.

22. Bao X, Zhang Q, Liu N, et al. Characteristics of circular RNA expression of pulmonary macrophages in mice with sepsis-induced acute lung injury. J Cell Mol Med 2019;23:7111-5.

23. Nie MW, Han YC, Shen ZJ, et al. Identification of circRNA and mRNA expression profiles and functional networks of vascular tissue in lipopolysaccharide-induced sepsis. J Cell Mol Med 2020;24:7915-27.

24. Zhang ZC, Guo XL, Li X. The novel roles of circular RNAs in metabolic organs. Genes Dis 2017;5:16-23.

25. Berezikov E, Guryev V, van de Belt J, et al. Phylogenetic shadowing and computational identification of human microRNA genes. Cell 2005;120:21-4.

26. Mitra A, Pfeifer K, Park KS. Circular RNAs and competing endogenous RNA (ceRNA) networks. Transl Cancer Res 2018;7:S624-S628.

27. Chen Q, Liu T, Bao Y, et al. CircRNA cRAPGEF5 inhibits the growth and metastasis of renal cell carcinoma via the miR-27a-3p/TXNIP pathway. Cancer Lett 2020;469:68-77.

28. Liao W, He J, Disoma C, et al. Hsa_circ_0107593 Suppresses the Progression of Cervical Cancer via Sponging hsa-miR-20a-5p/93-5p/106b-5p. Front Oncol 2021;10:590627.

29. D'Elia RV, Harrison K, Oyston PC, et al. Targeting the "cytokine storm" for therapeutic benefit. Clin Vaccine Immunol 2013;20:319-27.

30. Kawai T, Akira S. The role of pattern-recognition receptors in innate immunity: update on Toll-like receptors. Nat Immunol 2010;11:373-84.

31. Beltrán-García J, Osca-Verdegal R, Nacher-Sendra E, et al. Circular RNAs in Sepsis: Biogenesis, Function, and Clinical Significance. Cells 2020;9:1544.

32. Zhang C, Wang X, Chen Y, et al. The down-regulation of hsa_circ_0012919, the sponge for miR-125a-3p, contributes to DNA methylation of CD11a and CD70 in CD4(+) T cells of systemic lupus erythematous. Clin Sci
(Lond) 2018;132:2285-98.

33. Xiong J, Wang T, Tang H, et al. Circular RNA circMAN2B2 facilitates glioma progression by regulating the miR-1205/S100A8 axis. J Cell Physiol 2019;234:22996-3004.

34. Dubois C, Marce D, Faivre V, et al. High plasma level of S100A8/S100A9 and S100A12 at admission indicates a higher risk of death in septic shock patients. Sci Rep 2019;9:15660.

35. Denstaedt SJ, Spencer-Segal JL, Newstead MW, et al. S100A8/A9 Drives Neuroinflammatory Priming and Protects against Anxiety-like Behavior after Sepsis. J Immunol 2018;200:3188-200.

36. Liu S, Liu C, Wang Z, et al. microRNA-23a-5p acts as a potential biomarker for sepsis-induced acute respiratory distress syndrome in early stage. Cell Mol Biol 2016;62:31-7.

37. Zhang H, Caudle Y, Shaikh A, et al. Inhibition of microRNA-23b prevents polymicrobial sepsis-induced cardiac dysfunction by modulating TGIF1 and PTEN. Biomed Pharmacother 2018;103:869-78.

38. Levi M, van der Poll T. Inflammation and coagulation. Crit Care Med 2010;38:S26-34.

39. Schouten $M$, Wiersinga WJ, Levi $M$, et al. Inflammation, endothelium, and coagulation in sepsis. J Leukoc Biol 2008;83:536-45.

40. Chen Q, Yang Y, Hou J, et al. Increased gene copy number of DEFA1/DEFA3 worsens sepsis by inducing endothelial pyroptosis. Proc Natl Acad Sci U S A 2019;116:3161-70.

41. Cheng J, Liu Q, Hu N, et al. Downregulation of hsa circ_0068087 ameliorates TLR4/NF-kappaB/NLRP3 inflammasome-mediated inflammation and endothelial cell dysfunction in high glucose conditioned by sponging miR197. Gene 2019;709:1-7.

42. Li X, Liu CX, Xue W, et al. Coordinated circRNA Biogenesis and Function with NF90/NF110 in Viral Infection. Mol Cell 2017;67:214-227.e7.

(English Language Editor: R. Scott)

Cite this article as: Qi L, Yan Y, Chen B, Cao J, Liang G, Xu P, Wang Y, Ren Y, Mao G, Huang Z, Xu C, Jiang H. Research progress of circRNA as a biomarker of sepsis: a narrative review. Ann Transl Med 2021;9(8):720. doi: 10.21037/atm-21-1247 